\title{
Thermal Radiation, Chemical Reaction and Viscous Dissipation Effects on Unsteady MHD Flow of Viscoelastic Fluid Embedded in a Porous Medium
}

\author{
Binyam Zigta \\ College of Natural and Computational Science Debreberhan University, Ethiopia \\ tzigta@yahoo.com
}

\begin{abstract}
In this paper the effect of unsteady, incompressible, magneto hydrodynamics filled with electrically conducting viscoelastic fluid in an infinite vertical Couette porous channel wall embedded in a porous medium is analyzed. A uniform magnetic field is applied perpendicular to the channel wall. The temperature of the moving channel wall varies periodically and the temperature difference between the two infinite vertical channel walls is high due to thermal radiation. The Eckert number is the ratio of the kinetic energy of the flow to the temperature difference of the channel walls. The solution of the governing equations is obtained using regular perturbation techniques. These techniques are used to transform partial differential equations that are difficult to solve in closed form. These equations are reduced to a set of ordinary differential equations in dimensionless form so can be solved analytically. The effects of physical parameters Viz. Hartmann number, Viscoelastic parameter, Eckert number, Permeability of porous medium, Chemical reaction parameter, thermal Grashof number for heat transfer, modified Grashof number for mass transfer, frequency parameter and Schmidt number on flow parameters Viz., velocity, temperature and concentration has been discussed and shown graphically. The theoretical results have been supported by $M A T L A B$ code simulation study. The results show that velocity decreases with increasing values of frequency, Hartmann number and viscoelastic parameter but reverse effect is observed with temperature, thermal Grashof number, modified Grashof number and permeability of porous medium. Furthermore, The result shows that an increment in both thermal radiation parameter and Eckert number results in decrement of temperature near the moving porous channel wall while it approaches to a zero in the region close to the boundary layer of the stationary channel wall,. An increment in both chemical reaction and Schmidt number results in decreasing concentration. The velocity of fluid increases as Grashof number and modified Grashof number increases.
\end{abstract}

Keywords : Viscoelastic fluid; MHD; Couette channel walls; Permeability of porous medium

\section{Introduction}

The change of shear stress versus strain rate inside a fluid which depends on viscosity can be classified as a linear, nonlinear or plastic response. When the shear stress is linearly proportional to the strain rate it is known as Newtonian fluid, the constant of proportionality being the coefficient of dynamic viscosity. In case of non-Newtonian fluid the shear stress is nonlinear to strain rate, can even be time dependent and hence a constant coefficient of dynamic viscosity can't be defined. In non-Newtonian fluid flow the property is treated by rheology, the science is related to the deformation and flow of a substance. If viscosity decreases as the shear stress or strain rate remains constant i.e. depends on time such fluids which possess this property are Thixotropic. When shear stress is independent of strain rate the material possesses plastic deformation.

Viscoelastic is the property of materials which combines both viscous and elastic characteristics when undergoing deformation. The behavior of viscoelastic fluid is different from that of Newtonian and in elastic non-Newtonian fluids. This includes the presence of 
normal stresses in shear flows sensitive to deformation. Some examples which possess such properties are shampoo, hand cream, tooth paste, yoghurt, polymers and paint. The factors that affect the properties of viscoelastic are temperature, time and shear rate.

In fluid dynamics the word dissipation means conversion of energy from one form to other forms. The fluid flow requires kinetic energy and hence its velocity takes energy from the motion and transforms it into internal energy. The kinetic energy is dissipated, i.e., during the flow it is converted in to internal energy. The increment of the velocity of the fluid results in heating up of the fluid.

The cited process is irreversible in case of viscous fluid and is known as viscous dissipation. The characteristics of viscous dissipation effects depend on non-dimensional quantity Eckert number. Viscous dissipation is applied in geophysical flows and industries. The work done for deforming an elastic material is stored as potential energy which can be recovered mechanically. The dissipation function is always positive when applied to viscous fluid. But it may be either positive or negative when it is applied to elastic and viscoelastic materials.

Unsteady MHD flow with electrically conducting viscoelastic fluid embedded in a porous media are of great importance and have several applications in industry such as in polymer production, ceramics, lubrication, electromagnetic propulsion, which is closely associated with magneto-chemistry, requires a complete understanding of the equation of state shear stress-shear rate relationship, thermal conductivity and radiation, flow of nuclear fuel, flow of fluid metal and alloys.

From literature reviews it can be observed that there are very few papers which have been studied on unsteady MHD flow with electrically conducting viscoelastic fluid in vertical Couette channel walls embedded in a porous medium. The present study the author wants to fill the gap in which the effects of thermal radiation, chemical reaction, viscous dissipation, permeability of porous medium and electrically conducting viscoelastic fluid are considered.

Some of the literature reviews related to this study on MHD flow with electrically conducting viscoelastic fluid are mentioned as follows::Second order effects in elasticity, plasticity and fluid dynamics analyzed [1].The Magneto hydrodynamic free convection laminar flow of incompressible viscoelastic fluid was studied [2]. The MHD combined free and forced convection flow through two parallel porous walls studied [3]. An oscillatory mixed convection flow of electrically conducting viscoelastic fluid in an infinite vertical channel filled with porous medium analyzed [4]. MHD free convection flow of viscoelastic fluid past an infinite porous plate was investigated [5].The unsteady flow and heat transfer through an elastic viscous liquid along an infinite hot vertical porous moving plate with variable free stream and suction examined [6]. Flow and heat transfer of an electrically conducting viscoelastic fluid between two horizontal squeezing and stretching plates have been studied [7]. Heat and mass transfer in MHD viscoelastic fluid flow through a porous medium over a stretching sheet with chemical reaction studied [8]. MHD mixed convective viscoelastic fluid flow in a permeable vertical channel with Dufour effect and chemical reaction parameter studied [9]. Viscoelastic fluid flow past an infinite vertical porous plate in the presence of first order chemical reaction investigated [10]. MHD flow through a porous medium past a stretched vertical permeable surface in the presence of heat source or sink and a chemical reaction has been studied [11]. The three dimensional free convection flow through porous medium in a vertical channel with heat source and chemical reaction studied [12].The unsteady two dimensional flow and heat 
transfer through an elastic viscous liquid along an infinite hot vertical porous surface bounded by porous medium studied [13]. MHD flows in Non Newtonian fluids has been studied [16].

The effects of radiation on free convection MHD flow have attracted many scholars due to its applications in space science, this occurs by using high temperature and design of relevant equipment. Furthermore, heat and mass transfer with thermal radiation on free convection MHD flow are significant in the plate heat transfer. Recently, many scholars focus in this field of study due to its development in gas cooled nuclear reactors, nuclear power plants, gas turbines, space vehicles, and supersonic flights.

The unsteady convective flow in a moving plate with thermal radiation studied - 18].The combined effects of radiation and buoyancy force past a vertical plate has been studied [19]. The influence of thermal radiation on convective flows over a porous vertical plate analyzed [20].The effects of thermal radiation on heat and mass transfer over a moving vertical plate studied [21]. The effects of thermal radiation on mixed convection flow over a permeable vertical plate with magnetic field have been studied [22].

Studies of energy and concentration transfer due to chemical reaction have significant application in many branches of science and technology. The effects of concentration transfer with the involvement of chemical reaction are important in chemical process which helps to obtain high value products from cheaper raw materials. The quality of production and the flow behavior of the fluid is affected by the concentration goes through some kind of chemical reaction with the surrounding fluid.

The effects of mass transfer over a vertical oscillating plate with chemical reaction studied [23]. Chemical reaction and thermal radiation effects on MHD convective flow in a porous medium in the presence of suction studied [24]. The effects of magnetic field on MHD Couette flow of a third-grade fluid with chemical reaction studied [25].

Even though, the effects of thermal radiation, chemical reaction, viscous dissipation, permeability of porous medium and electrically conducting viscoelastic fluid in vertical Couette channel wall embedded in a porous medium are important they have not been considered in these studies [15]. Hence the author has been motivated to study the effects of thermal radiation, chemical reaction, viscous dissipation, permeability of porous medium and electrically conducting viscoelastic fluid embedded in a porous medium. The effect of MHD on free convection oscillatory Couette flow when the temperature and concentration oscillate with time in the presence of the thermal radiation and chemical reaction has been studied [28]. The effect of thermal radiation, chemical reaction and viscous dissipation on MHD flow has been studied [29]. The effects of thermal diffusion, magnetic field and viscous dissipation on unsteady mixed convection flow past a porous plate with chemical reaction has been investigated [30]. Radiation and chemical effects on MHD flow along a moving vertical porous plate [31]. Unsteady MHD free Convective Couette flow between vertical porous plates with thermal radiation studied [32].

The combined effect of magnetic field, thermal radiation; chemical reaction, viscous dissipation, permeability of porous medium and electrically conducting viscoelastic fluid between two vertical parallel Couette channel walls embedded in a porous medium will be considered and studied. The fluid considered here has the properties viz., unsteady, incompressible, electrically conducting viscoelastic, chemical reacting and thermal radiating. Free stream velocity of the fluid is assumed to be fluctuating with time. Furthermore, we assume temperature and concentration of the fluid are also fluctuating with time. 
The main objective of the present study is to analyze unsteady, incompressible, MHD flow with electrically conducting viscoelastic fluid on free convection oscillatory fluid flow bounded between two infinite vertical Couette channel walls embedded in a porous medium when the temperature and concentration oscillates with time in the presence of thermal radiation, chemical reaction, viscous dissipation and permeability of porous medium.

\section{Theoretical Review}

\subsection{Mathematical Model and Analysis of the Problem}

Consider two dimensional free convection Couette flows of unsteady, incompressible, MHD flow with electrical conducting viscoelastic fluid, chemical reacting and radiating fluid bounded by two infinite vertical channel walls separated by a distance $b$ immersed in a porous medium. The geometrical description of the model and coordinate system are shown in Figure 1. The $\bar{x}$ axis is parallel to the gravitational acceleration vector $g$ but in opposite direction. The $\bar{y}$ axis is transverse to the channel walls.

The vertical moving channel wall is located at $\bar{y}=0$ along $\bar{x}$ axis where the temperature is given by $\bar{T}_{m}$ and the concentration is also given by $\bar{C}_{m}$. The other stationary channel wall is located at $\bar{y}=b$ where the temperature is denoted by $\bar{T}_{b}$ and the concentration is denoted by $\bar{C}_{b}$.

Initially at time $\bar{t}=0$, the channel wall and the fluid are at the same temperature $\bar{T}_{b}$ in stationary position and with concentration level $\bar{C}_{b}$ at all points. At time $\bar{t}>0$, the channel wall temperature and concentration are raised $\bar{T}_{m}$ and $\bar{C}_{m}$ respectively and are maintained at a constant value. This result is due to porous medium i.e., porous medium is used to insulate a heated body by maintaining its temperature. As time changes temperature changes this leads to buoyancy force acting in fluid flow so the channel wall $\bar{y}=0$ starts moving in its own plane along positive $\bar{x}$ direction with velocity $V$. And for all the later time $\bar{t}>0$ the channel wall continues to be in motion. But due to viscosity of the fluid and acceleration due to gravity of the motion will decrease and consequently it returns after some time $\bar{t}$. The moving channel wall is being heated by supplying of heat so as to maintain its temperature constant, while the stationary channel wall $\bar{y}=b$ is maintained at the constant temperature $\bar{T}_{b}$.

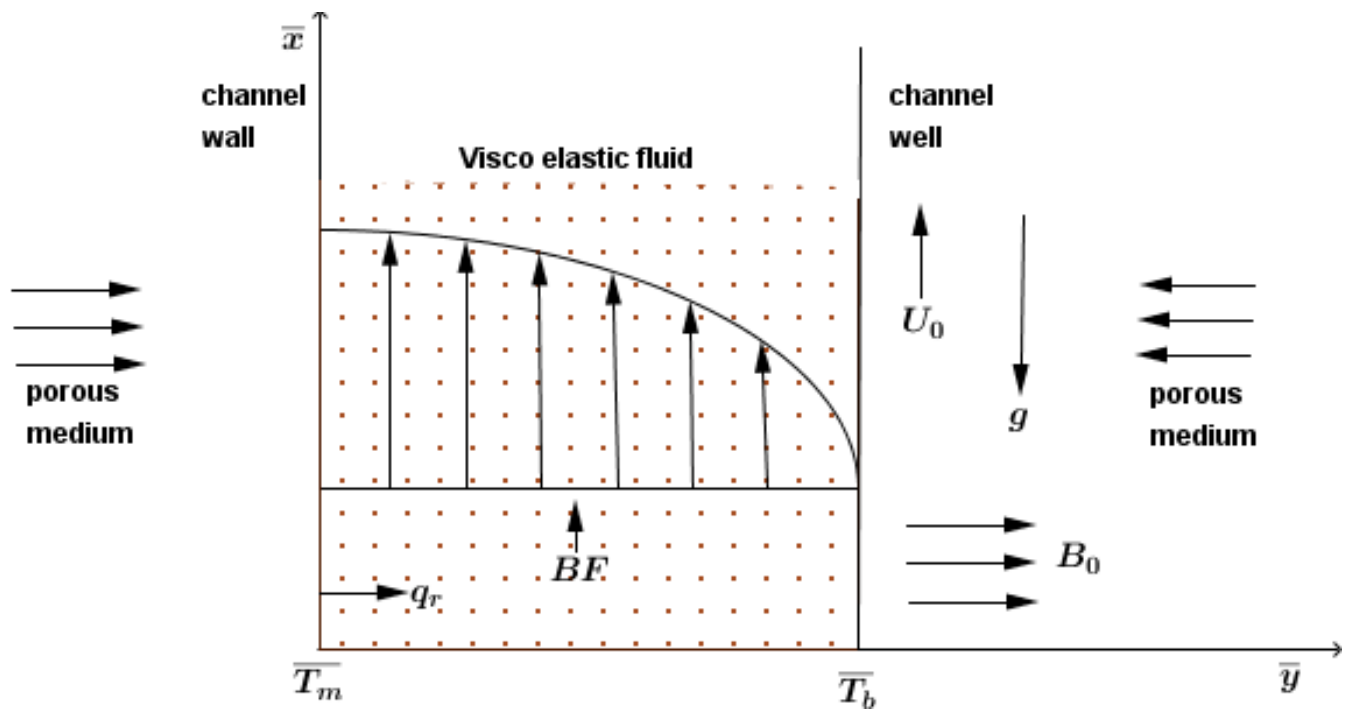

Figure 1. Flow configuration of the model problem 
It is assumed that the radiation heat flux is to be present in the form of unidirectional flux in $\bar{y}$ direction. We will derive the governing equations of the model by assuming the following: The fluid properties such as temperature, viscosity, pressure, specific volume, specific gravity are constant; the influence of the density variation with both temperature and concentration in the body force term or Boussinesq's approximation is not constant; the flow of the fluid is unsteady, laminar, incompressible, electrically conducting viscoelastic, thermal radiating and chemical reacting; the magnetic field applied is transversal between the channel walls; the induced magnetic field of the fluid is negligible and hence the magnetic Reynolds number is a very small constant, the external electric field is supposed to be zero; the electric field due to polarization of charges is negligible; viscosity is also considered with the constant permeability of porous medium; we assume that there exist a homogeneous chemical reaction of first order with constant rate between diffusing concentration or species and the fluid in the moving plate A reaction is said to be of first order if the rate of reaction is directly proportional to concentration.

We assume that the free stream velocity follows according to the expression

$$
\bar{U}(\bar{t})=U_{o}\left(1+\varepsilon e^{i \bar{\omega} \bar{t}}\right) .
$$

Here in (1), $U_{o}$ represents the mean constant free stream velocity, $\bar{\omega}$ is the frequency and $\bar{t}$ is the time.

We now derive the governing equations of the present model:

The momentum equation of the model takes the form as

$$
\frac{\partial \bar{u}}{\partial \bar{t}}=\frac{\partial \bar{U}}{\partial \bar{t}}+v \frac{\partial^{2} \bar{u}}{\partial \bar{y}^{2}}+g \beta\left(\bar{T}-\bar{T}_{b}\right)+g \beta_{c}\left(\bar{C}-\bar{C}_{b}\right)-\frac{J X B}{\rho}-\frac{v \bar{u}}{\bar{k}}+\frac{\bar{k}_{o}}{\rho} \frac{\partial \tau_{x y}}{\partial \bar{y}} .
$$

In (2), the vector cross product $J x B$ represents the Lorentz force. This term is a body force corresponding to magneto hydrodynamics flow. The total magnetic field is represented by $B$. The density of the current is represented by $J, \bar{k}$ denotes the permeability of porous medium and $\bar{k}_{o}$ is the viscoelastic parameter.

Using ohm's law, the expression for the density of current $J$ can be constructed as

$$
J=\sigma(E+\bar{v} x B) .
$$

Here in (3), $\sigma$ is the electric conductivity, $E$ is the electric field and $\bar{v}$ is the velocity of the fluid. Upon substituting $E=0$ in (3) it reduces to

$J=\sigma(\bar{v} \times B)$.

Also, the expression for Lorentz force reduces and takes the form as

$J x B=-\sigma B^{2} \bar{u}$.

In view of the result (5), the equation (2) reduces to

$$
\frac{\partial \bar{u}}{\partial \bar{t}}=\frac{\partial \bar{U}}{\partial \bar{t}}+v \frac{\partial^{2} \bar{u}}{\partial \bar{y}^{2}}+g \beta\left(\bar{T}-\bar{T}_{b}\right)+g \beta_{c}\left(\bar{C}-\bar{C}_{b}\right)-\frac{\sigma B^{2}(\bar{u}-\bar{U})}{\rho}-\frac{v \bar{u}}{\bar{k}}+\frac{\bar{k}_{o}}{\rho} \frac{\partial \tau_{x y}}{\partial \bar{y}} .
$$

The energy equation of the model can be expressed as

$$
\frac{\partial \bar{T}}{\partial \bar{t}}=\alpha \frac{\partial^{2} \bar{T}}{\partial \bar{y}^{2}}-\frac{1}{\rho C_{p}} \frac{\partial q_{r}}{\partial y}+\left(\frac{\mu}{\rho C_{p}}\right)\left(\frac{\partial \bar{u}}{\partial \bar{y}}\right)^{2} .
$$

The concentration equation of the model can be expressed as 


$$
\frac{\partial \bar{C}}{\partial \bar{t}}=D \frac{\partial^{2} \bar{C}}{\partial \bar{y}^{2}}-k_{r}\left(\bar{C}-\bar{C}_{b}\right) .
$$

In (6) $\tau_{x y}$ is the component of the shear stress of the viscoelastic fluid given in [26] as,

$$
\tau_{x y}=\mu \frac{\partial \bar{u}}{\partial \bar{y}}-\frac{\mu}{\alpha^{*}} \frac{\partial \tau_{x y}}{\partial \bar{t}} .
$$

Here $\mu$ is the coefficient of dynamic viscosity and $\alpha^{*}$ is the modulus of rigidity. If $\alpha^{*}$ approaches to infinity or if the component of shear stress is independent of time that is at steady state, the fluid behaves like a viscous fluid without elasticity. Solving (9) for $\tau_{x y}$ in terms of the velocity component $\bar{u}$ we obtain

$\frac{\partial \tau_{x y}}{\partial \bar{y}}=\frac{\partial}{\partial \bar{y}}\left(\mu \frac{\partial \bar{u}}{\partial \bar{y}}\right)-\frac{1}{\alpha^{*}} \frac{\partial}{\partial \bar{y}}\left(\mu \frac{\partial}{\partial \bar{t}}\left(\mu \frac{\partial \bar{u}}{\partial \bar{y}}\right)\right) \approx \mu \frac{\partial^{2} \bar{u}}{\partial \bar{y}^{2}}-\frac{\mu^{2}}{\alpha^{*}} \frac{\partial^{3} \bar{u}}{\partial \bar{t} \partial \bar{y}^{2}}$.

The term $\left[\left(1 / \alpha^{* 2}\right) \frac{\partial}{\partial \bar{y}}\left(\mu \frac{\partial}{\partial \bar{t}}\left(\mu \frac{\partial \tau_{x y}}{\partial \bar{y}}\right)\right)\right]$ is neglected if $\alpha^{*}$ approaches to infinity. Then substituting (10) into (2) and after some algebraic manipulations we obtain

$$
\omega \frac{\partial u}{\partial t}=\omega \frac{\partial U}{\partial t}+\left(1+\frac{k_{0} v \mu}{U_{0}{ }^{2}}\right) \frac{\partial^{2} u}{\partial y^{2}}+G r \theta+G c C-M^{2}(u-U)-\frac{U_{0}^{2} u b^{2}}{k v^{2}}-\frac{k_{0} v^{2} \mu^{2} \omega}{U_{0}{ }^{2} \alpha^{*} b^{2}} \frac{\partial^{3} u}{\partial t \partial y^{2}} .
$$

The equations (6) - (8) govern the present model described in Figure 1. Here in (6) - (8) we have used some parameters which can be assigned physical interpretations as follows: $\alpha$ is thermal diffusivity; $\rho$ is density of the fluid; $g$ is the acceleration due to gravity; $\beta$ is the thermal expansion coefficient, $\beta_{c}$ the concentration expansion coefficient; $\bar{T}$ is the temperature of the fluid in the boundary layer; $\bar{T}_{m}$ is the temperature of the moving channel wall; $\bar{T}_{b}$ is the temperature of the stationary channel wall; $q_{r}$ is the radiative heat flux; $D$ is the mass diffusivity; $\bar{k}$ is the permeability of porous medium, $\bar{k}_{0}$ is the viscoelastic parameter. The term $\left[\left(1 / \rho C_{p}\right)\left(\partial q_{r} / \partial y\right)\right]$ is the thermal radiation effect; and $k_{r}\left(\bar{C}-\bar{C}_{b}\right)$ is the generative chemical reaction.

The physical interpretation of the minus sign in (6) shows that the fluid flows in the direction from higher to lower potential and the plus sign in shear stress of viscoelastic fluid is tensile stress or pulling force.. In (7), the minus sign of the radiative heat flux term $\left[\left(1 / \rho C_{p}\right)\left(\partial q_{r} / \partial y\right)\right]$ indicates the emission of energy away from the objects that is heat is lost. Furthermore, in the energy equation (7), $\alpha>0$ shows heat generation and $\alpha<0$ indicates heat absorption i.e., thermal energy diffuses more rapidly through substances with high thermal diffusivity $\alpha$ and it diffuses slowly through those with low thermal diffusivity $\alpha$. The plus sign of the term $\left(\frac{\mu}{\rho C_{p}}\right)\left(\frac{\partial \bar{u}}{\partial \bar{y}}\right)^{2}$ indicates it is applied to viscous fluid.

In (8), the minus sign indicates that the mass diffusion represents 'Generative chemical reaction'. Here chemical reaction is an exothermic reaction and in that case heat is generated. However, the chemical reaction is said to be generative or exothermic if the chemical reaction parameter is negative i.e. $k_{r}<0$.If $k_{r}>0$ the chemical reaction is said to be destructive or endothermic chemical reaction 
Based on Figure 1 the boundary conditions of the model (6) - (8) can be expressed as:

$$
\begin{gathered}
\bar{y}=0, \bar{u}=U_{0}\left(1+\varepsilon e^{i \bar{\omega} \bar{t}}\right), \\
\bar{T}=\bar{T}_{m}+\underset{(12)}{\varepsilon\left(\bar{T}_{m}-\bar{T}_{b}\right) e^{i \bar{\omega} \bar{t}},} \\
\bar{C}=\bar{C}_{m}+\varepsilon\left(\bar{C}_{m}-\bar{C}_{b}\right) e^{i \overline{\bar{\omega}} \bar{t}},
\end{gathered}
$$

$\bar{y}=b, \quad \bar{u}=0, \bar{T}=\bar{T}_{b}, \quad \bar{c}=\bar{c}_{b}$.

Using the Roseland approximation for radiative heat transfer and the Roseland approximation for diffusion and also following the other scholarly works [27], the expression for radiative heat flux $q_{r}$ can be given as

$$
q_{r}=\left(\frac{-4 \sigma}{3 k_{s}}\right)\left(\frac{\partial \bar{T}^{4}}{\partial \bar{y}}\right)
$$

Here in (16), the parameters $\sigma$ and $k_{s}$ represent the Stefan Boltzmann constant and the Roseland mean absorption coefficient respectively.

We now assume that the temperature differences within the fluid flow are sufficiently small so that $\bar{T}^{4}$ in (16) can be expressed as a linear function of $\bar{T}_{b}$ using Taylor series expansion. The Taylor series expansion of $\bar{T}^{4}$ about $\bar{T}_{b}$, after neglecting the higher order terms, takes the form as

$$
\bar{T}^{4} \cong 4 \bar{T}_{b}^{3} \bar{T}^{-3} \bar{T}_{b}^{4}
$$

Using (16) and (17) in (7), we obtain

$$
\frac{\partial \bar{T}}{\partial \bar{t}}=\alpha\left(\frac{\partial^{2} \bar{T}}{\partial \bar{y}^{2}}\right)+\left(\frac{1}{\rho C_{p}}\right)\left(\frac{16 \sigma \bar{T}_{b}{ }^{3}}{3 k_{s}}\right)\left(\frac{\partial^{2} \bar{T}}{\partial \bar{y}^{2}}\right)+\left(\frac{Q_{0}}{\rho C_{p}}\right)\left(\bar{T}-\bar{T}_{b}\right)+\left(\frac{\mu}{\rho C_{p}}\right)\left(\frac{\partial \bar{u}}{\partial \bar{y}}\right)^{2} .
$$

In Eq. (18), the term $\left[\left(Q_{0} / \rho C_{p}\right)\left(\bar{T}-\bar{T}_{b}\right)\right]$ denotes heat absorption of the fluid and $\left(\frac{\mu}{\rho C_{p}}\right)\left(\frac{\partial \bar{u}}{\partial \bar{y}}\right)^{2}$ denotes viscous dissipation.

\subsection{Non Dimensionalization of the Model}

In order to solve the governing equations (6) - (8) of the model it is convenient and easy to deal with its dimensionless form. Hence, we find the dimensionless form of the model by introducing the following non-dimensional quantities

$$
\begin{gathered}
y=\bar{y} / b, \quad R=4 \sigma T_{b}{ }^{3} / \kappa k_{s}, \quad u=\bar{u} / U_{0}, \quad U=\bar{U} / U_{0}, \quad t=\bar{\varpi} \bar{t}, \\
\varpi=\bar{\varpi} b^{2} / v,
\end{gathered}
$$




$$
\begin{aligned}
& G c=\left\{\left[g \beta_{c} b^{2}\left(\bar{C}_{m}-\bar{C}_{b}\right)\right] / v U_{0}\right\}, G r=\left\{g \beta b^{2}\left(\bar{T}_{m}-\bar{T}_{b}\right) / v U_{0}\right\}, \quad S c=v / D, \quad \operatorname{Pr}=v / \alpha, \\
& M=\sqrt{\left(\sigma B^{2} b^{2} / \rho v\right)}, C=\left[\left(\bar{C}-\bar{C}_{b}\right) /\left(\bar{C}_{m}-\bar{C}_{b}\right)\right], \quad k=\bar{k} U_{0}{ }^{2} / v^{2}, \quad k_{o}=\frac{\bar{k}_{o} U_{0}{ }^{2}}{\rho v^{2}} \\
& \theta=\left[\left(\bar{T}-\bar{T}_{b}\right) /\left(\bar{T}_{m}-\bar{T}_{b}\right)\right], E c=U_{o}{ }^{2} / C_{\mathrm{p}} \Delta T ; \Delta T=T_{w}^{\prime}-T_{h}^{\prime}
\end{aligned}
$$

After substituting the above non-dimensional quantities in (6) - (8) and after simple algebraic manipulations, the non-dimensional form of the model takes the following form as

$$
\begin{aligned}
& \omega \frac{\partial u}{\partial t}=\omega \frac{\partial U}{\partial t}+\left(1+\frac{k_{0} v \mu}{U_{0}^{2}}\right) \frac{\partial^{2} u}{\partial y^{2}}+G r \theta+G c C-M^{2}(u-U)-\frac{U_{0}^{2} u b^{2}}{k v^{2}}-\frac{k_{0} v^{2} \mu^{2} \omega}{U_{0}^{2} \alpha b^{2}} \frac{\partial^{3} u}{\partial t \partial y^{2}} \\
& \omega \frac{\partial \theta}{\partial t}=\frac{1}{p r}\left(1+\frac{4 R}{3}\right) \frac{\partial^{2} \theta}{\partial y^{2}}+\frac{Q_{o}}{\rho C_{p} v} \theta h^{2}+E c\left(\frac{\partial u}{\partial y}\right)^{2} \\
& \omega S c \frac{\partial C}{\partial t}=\frac{\partial^{2} C}{\partial y^{2}}-\frac{K_{r} C h^{2}}{D} .
\end{aligned}
$$

The corresponding boundary conditions (12) - (15) take the form as

$$
\begin{aligned}
& y=0, u=1+\varepsilon e^{i t}, \theta=1+\varepsilon e^{i t}, C=1+\varepsilon e^{i t}, \\
& y=1, u=0, \theta=0, C=0 .
\end{aligned}
$$

The system of equations (19)-(21) together with the boundary conditions (22) - (23) constitutes the non-dimensional form of the present model.

\section{Discussion}

\subsection{Analytical Solution to the problem}

To find the analytical solution of the non dimensional form of the present model we consider equations (19) - (23) and we assume the amplitudes of the free stream velocity, temperature and concentration variation are very small quantities. Hence, the amplitude of free stream velocity needs to be considered $0<\varepsilon<1$

Using perturbation techniques the solution of the model has the following form

$$
\begin{aligned}
& u(y, t)=u_{0}(y)+\varepsilon u_{1}(y) e^{i t} \\
& \theta(y, t)=\theta_{0}(y)+\varepsilon \theta_{1}(y) e^{i t} \\
& C(y, t)=C_{0}(y)+\varepsilon C_{1}(y) e^{i t}
\end{aligned}
$$

Also, the free stream velocity takes the form as

$$
U=1+\varepsilon e^{i t}
$$

The partial differential equations (19) - (21) cannot be solved in closed form or explicit form so it is solved analytically after these equations are reduced to a set of ordinary differential equations in dimensionless form. 
On substituting equations (24) - (27) into (19) - (23), and equating harmonic and non-harmonic terms and neglecting higher orders of $\varepsilon$ we obtain the following system of equations:

$u_{0}^{\prime \prime \prime}-\phi u_{0}^{\prime \prime}+\psi=\left[G r \theta_{0}+G c C_{0}-M^{2}\left(u_{0}-1\right)\right] \chi$

$u_{1}{ }^{\prime \prime \prime}-\Omega u_{1}^{\prime \prime}+\Gamma=\left[G r \theta_{1}+G c C_{1}-\left(M^{2}+i \omega\right)\left(u_{1}-1\right)\right] \mathrm{K}$

$\frac{1}{P r}\left(1+\frac{4 R}{3}\right) \theta_{0}^{\prime \prime}+\frac{Q_{0} \theta_{0} h^{2}}{\rho C_{p} v}=-E c u_{0}^{\prime 2}$

$\frac{1}{P r}\left(1+\frac{4 R}{3}\right) \theta_{1}^{\prime \prime}+\left(\frac{Q_{0} h^{2}}{\rho C_{p} v}-i \omega\right) \theta_{1}=-2 E c u_{0}^{\prime} u_{1}^{\prime}$

$C_{0} "-\frac{K_{r} C_{o} h}{D}=0$

$C_{1} "-C_{1}\left(\frac{K_{r} h^{2}}{D}+i \omega S c\right)=0$

Further, the new boundary conditions corresponding to (22) - (23) obtained after non dimensionalization as

$y=0, u_{0}=1, u_{1}=1, \theta_{0}=1, \theta_{1}=1, C_{0}=1, C_{1}=1$

$y=1, u_{0}=0, u_{1}=0, \theta_{0}=0, \theta_{1}=0, C_{0}=0, C_{1}=0$

The variables $\theta_{0}, \theta_{1}, C_{0}$ and $C_{1}$ are still coupled in(28) - (33). To solve (28) - (33) it is proposed that the Eckert number $E c$ is very small for incompressible fluid and assume that

$$
F(y)=F_{0}(y)+E c F_{1}(y)+o\left(E c^{2}\right) .
$$

Here $F$ stands for any variable $\theta_{0}, \theta_{1}, C_{0}$ and $C_{1}$. These variables can be expanded in powers of $E c$ as follows:

$\theta_{0}(y)=\theta_{00}(y)+E c \theta_{01}(y)$

$\theta_{1}(y)=\theta_{10}(y)+E c \theta_{11}(y)$

$C_{0}(y)=C_{00}(y)+E c C_{01}(y)$

$C_{1}(y)=C_{10}(y)+E c C_{11}(y)$

Substituting (36) - (39) in to (28) - (33) and equating terms free from $E c$ and with coefficients $E c$ and neglecting higher orders of $E c$ the following equations are obtained:

$C^{\prime \prime}{ }_{00}-\frac{k_{r} C_{00} h^{2}}{D}=0$

$C^{\prime \prime}{ }_{01}-\frac{k_{r} C_{01} h^{2}}{D}=0$

$C_{10}^{\prime \prime}-C_{10}\left[\frac{k_{r} h^{2}}{D}+i \omega S c\right]=0$

$C_{11}^{\prime \prime}-C_{11}\left[\frac{k_{r} h^{2}}{D}+i \omega S c\right]=0$ 


$$
\begin{aligned}
& \frac{1}{P r}\left(1+\frac{4 R}{3}\right) \theta_{00}^{\prime \prime}+\frac{Q_{0} \theta_{00} h^{2}}{\rho C_{p} v}=0 \\
& \frac{1}{P r}\left(1+\frac{4 R}{3}\right) \theta_{01}^{\prime \prime}+\frac{Q_{0} \theta_{01} h^{2}}{\rho C_{p} v}=-u_{00}^{\prime 2} \\
& \frac{1}{P r}\left(1+\frac{4 R}{3}\right) \theta_{10}^{\prime \prime}+\left[\frac{Q_{0} h^{2}}{\rho C_{p} v}-i \omega\right] \theta_{10}=0 \\
& \frac{1}{P r}\left(1+\frac{4 R}{3}\right) \theta_{11}^{\prime \prime}+\left[\frac{Q_{0} h^{2}}{\rho C_{p} v}-i \omega\right] \theta_{11}=-2 u_{00}^{\prime} u_{10}^{\prime}
\end{aligned}
$$

Equations (40) - (47) are subjected to the new boundary conditions as given below:

$y=0, u_{00}=u_{10}=\theta_{00}=\theta_{10}=C_{00}=C_{10}=1, u_{01}=u_{11}=\theta_{01}=\theta_{11}=C_{01}=C_{11}=0$

$y=1, u_{00}=u_{01}=u_{10}=u_{11}=\theta_{00}=\theta_{01}=\theta_{10}=\theta_{11}=C_{00}=C_{01}=C_{10}=C_{11}=0$

Solving equations (40) - (47) together with the boundary conditions (48) - (49), the analytical solutions are obtained:

$$
\begin{aligned}
\theta_{00} & =c_{1} e^{\lambda y}+c_{2} e^{-\lambda y} \\
\theta_{01} & =c_{1}^{\prime} e^{\lambda y}+c_{2}^{\prime} e^{-\lambda y}=0 \\
\theta_{10} & =k_{1} e^{\mu y}+k_{2} e^{-\mu y} \\
\theta_{11} & =k_{1}^{\prime} e^{\mu y}+k_{2}^{\prime} e^{-\mu y}=0 \\
C_{00} & =d_{1} e^{m y}+d_{2} e^{-m y} \\
C_{01} & =f_{1} e^{m y}+f_{2} e^{-m y}=0 \\
C_{10} & =h_{1} e^{\gamma y}+h_{2} e^{-\gamma y} \\
C_{11}= & p_{1} e^{\gamma y}+p_{2} e^{-\gamma y}=0
\end{aligned}
$$

Also $u(y, t), \theta(y, t)$ and $C(y, t)$ are given by

$$
u(y, t)=A e^{M y}+B e^{-M y}+\frac{G r \theta_{00}+G c C_{00}+M^{2}}{M^{2}}+\varepsilon\left(c e^{\beta y}+d e^{-\beta y}+\frac{G r \theta_{10}+G c C_{10}}{M^{2}}\right) e^{i t}
$$

$$
\begin{aligned}
& \theta(y, t)=c_{1} e^{\lambda y}+c_{2} e^{-\lambda y}+\varepsilon\left(k_{1} e^{\mu y}+k_{2} e^{-\mu y}\right) e^{i t} \\
& C(y, t)=d_{1} e^{m y}+d_{2} e^{-m y}+\varepsilon\left(h_{1} e^{\gamma y}+h_{2} e^{-\gamma y}\right) e^{i t}
\end{aligned}
$$

Various symbols used here and above are expressed in the APPENDIX. 


\subsection{Simulation study of the problem}

This section analyzes simulation of different flow parameters using MATLAB code. Consider an unsteady incompressible, magneto hydrodynamics with electrically conducting viscoelastic fluid on free convection oscillatory fluid flow bounded between two infinite vertical Couette channel walls embedded in a porous medium when the temperature and concentration oscillates with time in the presence of thermal radiation, chemical reaction, viscous dissipation and permeability of porous medium. For the sake of simplicity we have not presented the graphs of skin friction, Nusselt number and Sherwood number for velocity, temperature and concentration profiles respectively.

The effects of physical parameters such as viscoelastic parameter, permeability of porous medium, thermal Grashof number, modified Grashof number, Hartmann number, frequency parameter, radiation parameter, chemical reaction parameter and Schmidt number on flow variables such as velocity, temperature, concentration of the model have been studied using simulation.

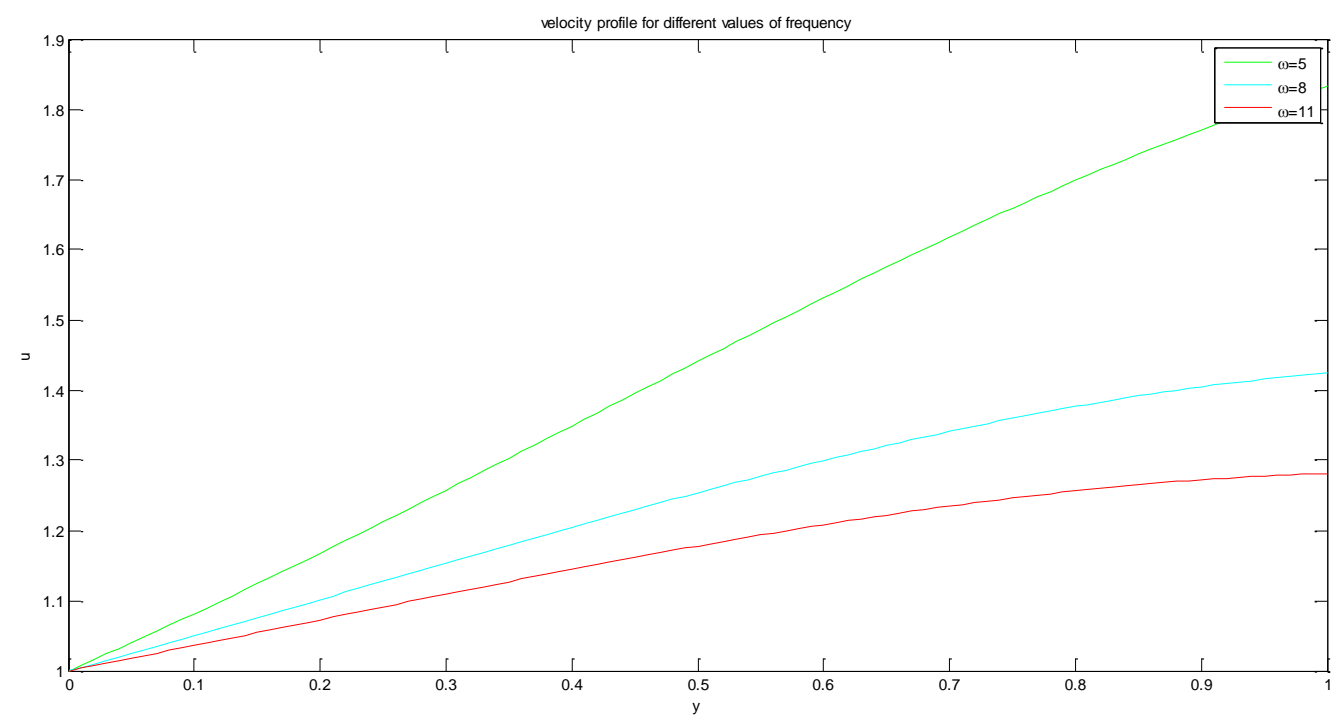

Figure 2. Velocity profile of the model for different values of frequency

In Figure 2, the simulated results of the influence of frequency parameter on velocity have been presented. The graph is plotted $y$ the distance between the channel walls against velocity $u$ holding other parameters constant. From the figure we observed that the minimum velocity $u$ is attained near the moving channel wall and then it increases as it goes to the stationary channel wall. Furthermore, we observed that the velocity decreases with increasing values of frequency. 


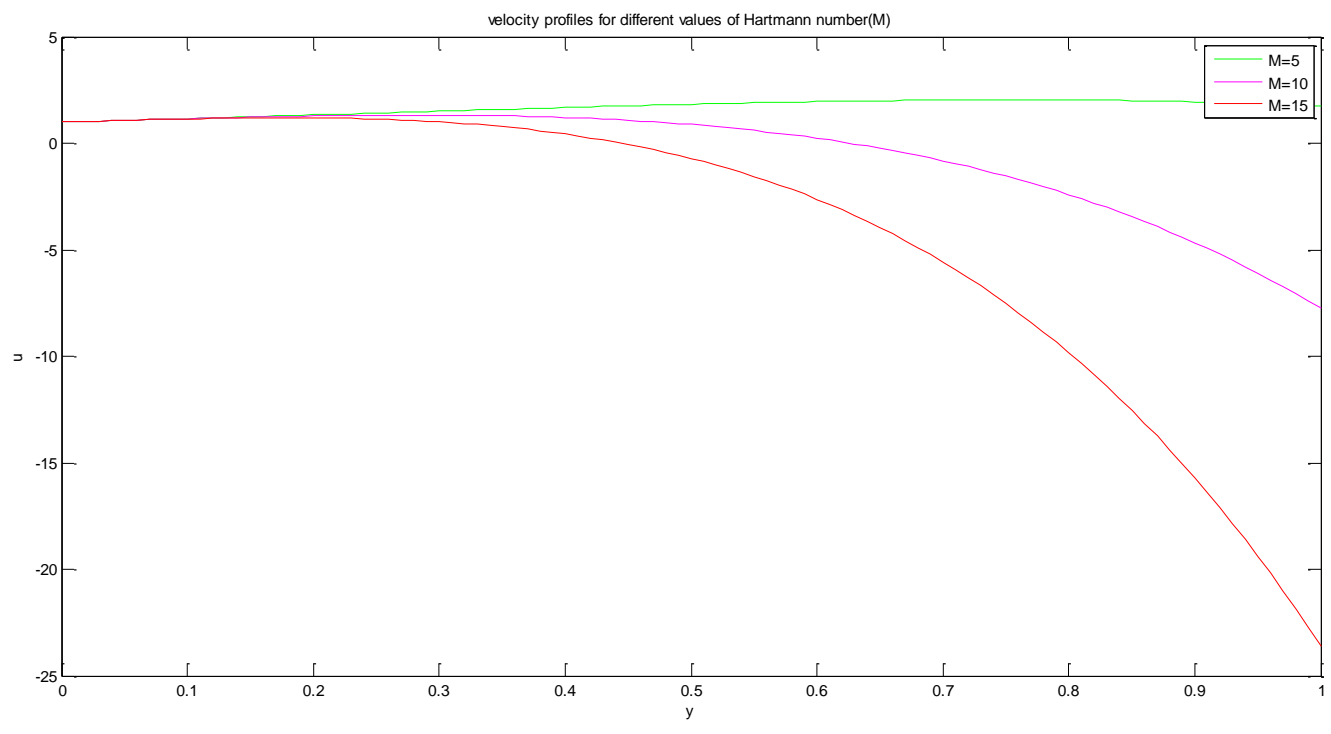

Figure 3. Velocity profile of the model for different values of Hartmann number

Figure 3, the simulated results of the Hartmann number on velocity presented. The graph is drawn $y$ the distance between the channel walls against velocity $u$ holding other parameters constant. From the figure it can be observed if the value of Hartmann number $M$ is increased more than the strength of resistance of the magnetic field of the fluid flow will also increase more; and as a result the velocity also will decrease more. Furthermore, the decrement in velocity results in decreasing the temperature. It can be put in simple words that whenever the Hartmann number increases then the velocity as well as thermal boundary layer will decrease.

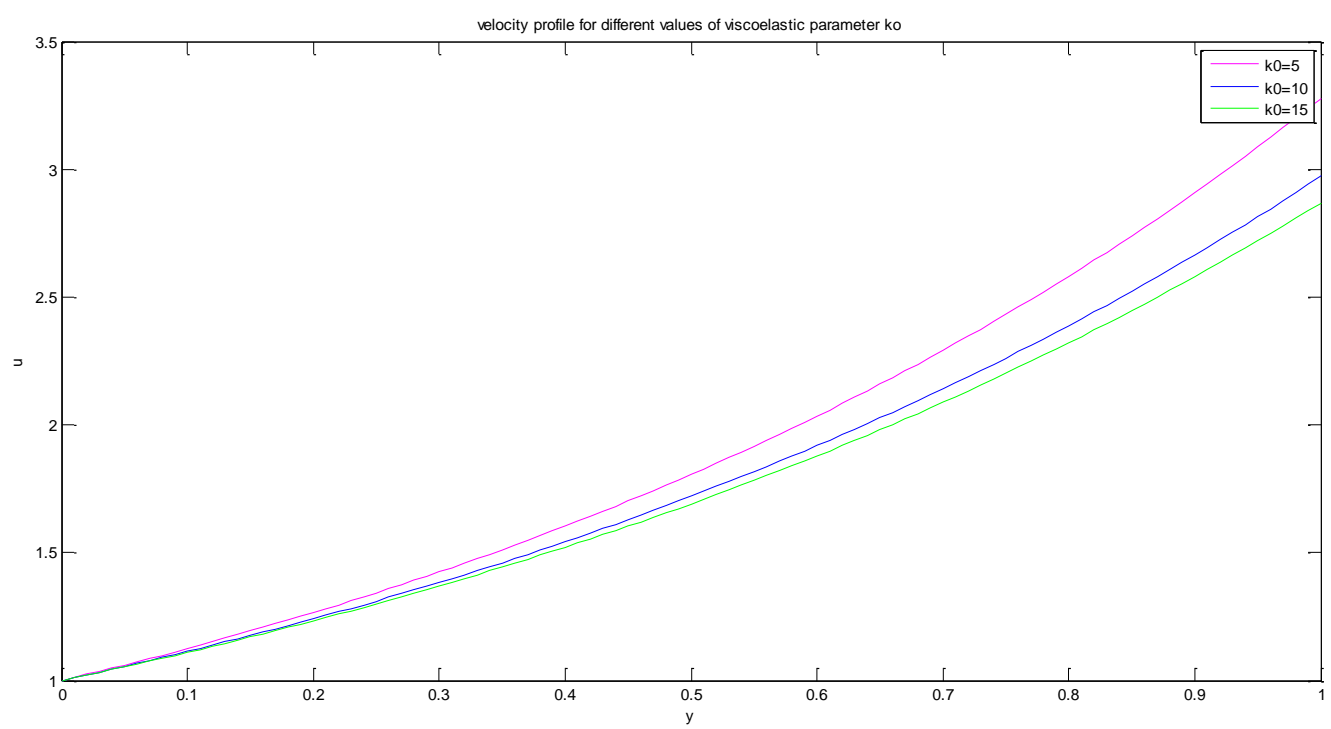

Figure 4. Velocity profile of the model for different values of viscoelastic parameter 
Figure 4, shows the influence of viscoelastic parameter $k_{0}$ on velocity profile of fluid while holding the other parameters constant. The graph of the velocity value is plotted by taking the distance from moving channel wall to stationary channel wall denoted by $y$ along the horizontal axis while taking the value of the fluid velocity denoted by $u$ along vertical axis.

The result shows that for a fixed value of viscoelastic parameter $k_{0}$, the value of the fluid velocity denoted by $u$ starts from a minimum constant value at the moving plate, and as $y$ increases the velocity $u$ increases and reaches a maximum constant value at the stationary channel wall. Generally, we can conclude from the simulation study that as viscoelastic parameter $k_{0}$ increases the velocity profile decreases i.e. flow of the fluid retards with increasing of viscoelastic parameter.

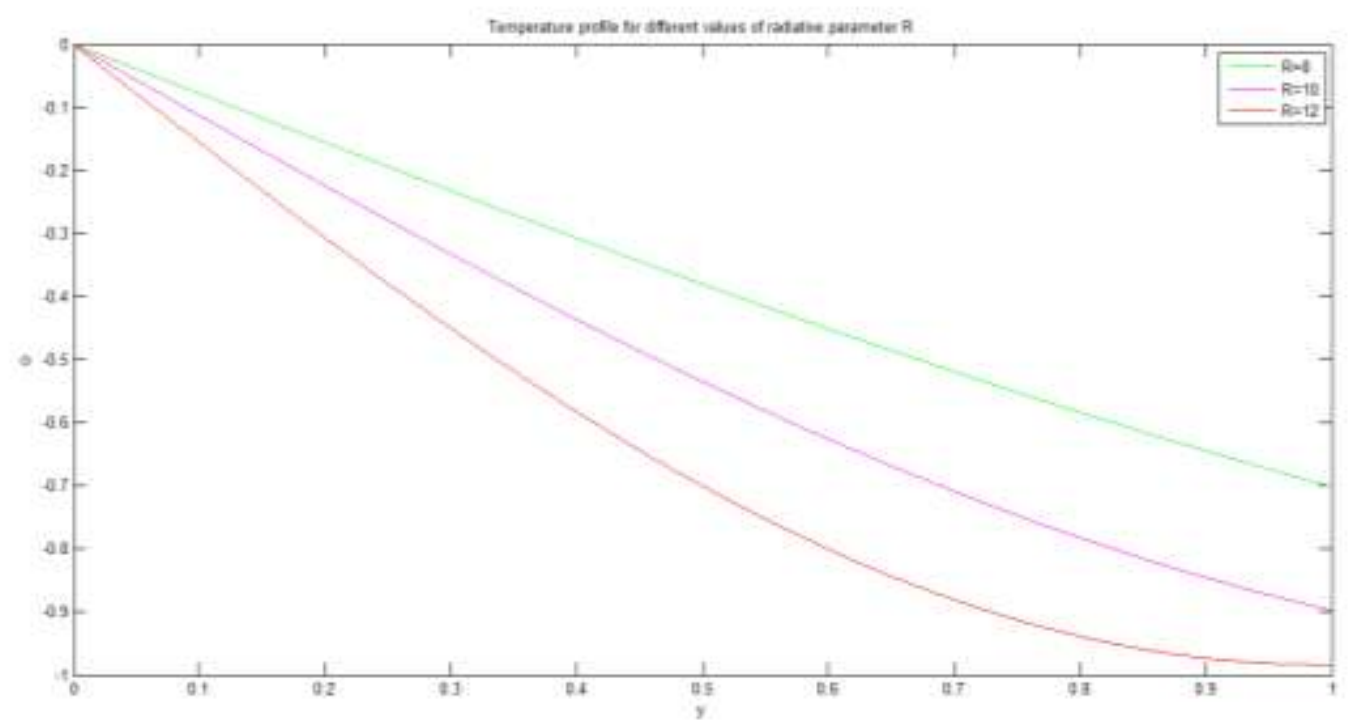

Figure 5. Temperature profile of the model for different values of the radiative parameter

In Figure 5, the simulated result of the influence of the radiation parameter on transfer of the temperature is presented. The graph is drawn in $y \theta$ plane representing respectively the distance between the channel walls and temperature. Other parameters are held constant. The result shows that for a fixed value of the thermal radiation parameter, the temperature $\theta$ starts from a constant value at the moving channel wall. Also as $y$ increases the temperature decreases till it reaches a minimum value. However, thereafter the temperature decreases with the increase of $y$ and ultimately the temperature reaches a lower constant value. Nevertheless, the measure of temperature at the moving channel wall is always at a higher value than that at the stationary channel wall. 


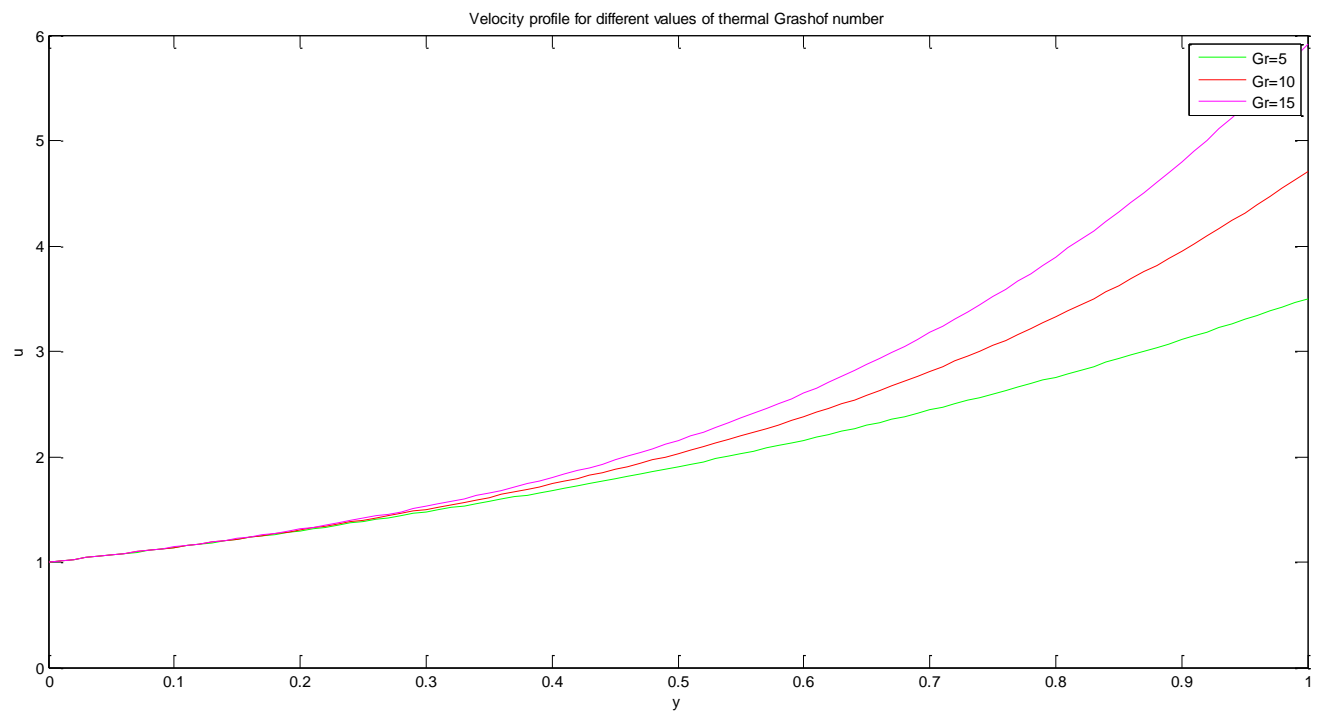

Figure 6. Velocity profile of the model for different values of thermal Grashof number

Figure 6, shows the influence of thermal Grashof number on velocity profile of the fluid while holding the other parameters constant. The graph of the velocity value is plotted by taking the distance from moving channel wall to stationary channel wall denoted by $y$ along the horizontal axis while taking the value of the fluid velocity denoted by $u$ along vertical axis.

The result shows that for a fixed value of thermal Grashof number, the value of the fluid velocity denoted by $u$ starts from a minimum constant value at the moving channel wall, and as $y$ increases the velocity $u$ increases and reaches a maximum constant value at the stationary channel wall. Generally, we can conclude from the simulation study that as thermal Grashof number increases the velocity profile increases. Physically, this means that the increment of the buoyancy force leads to increase the vertical component of the velocity.

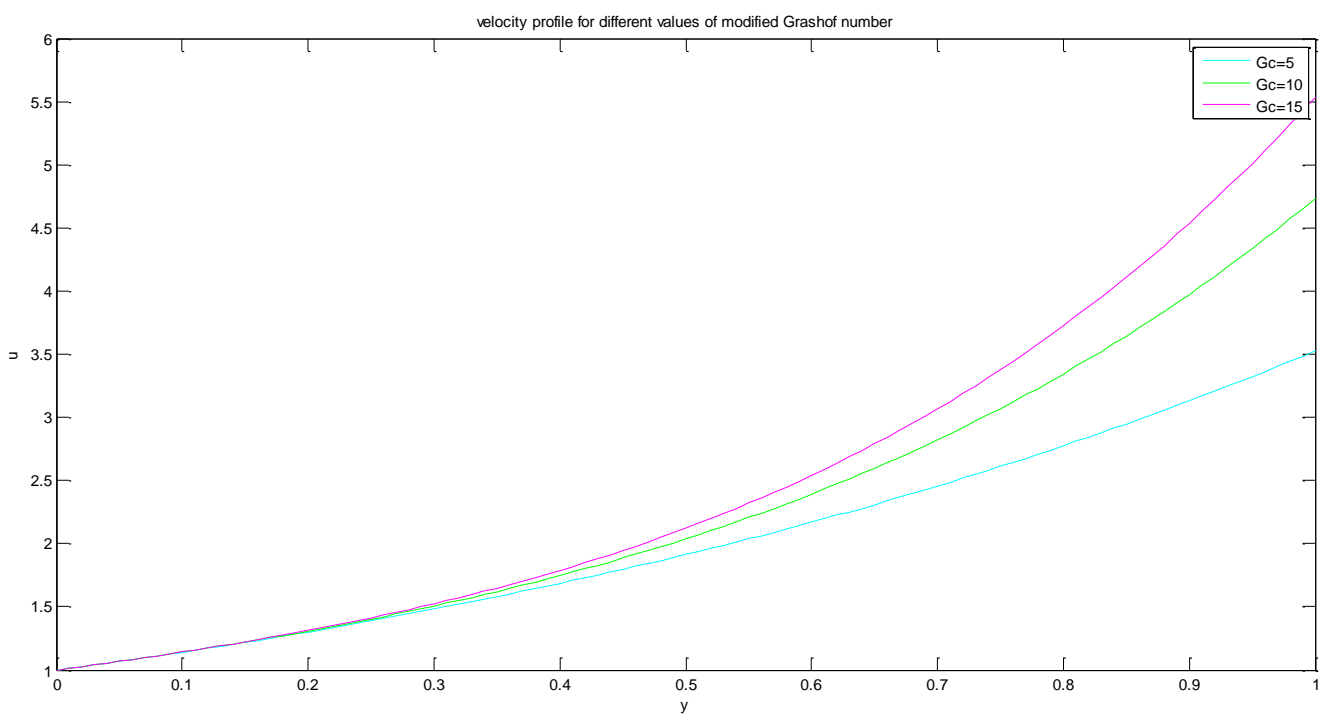

Figure 7. Velocity profile of the model for different values of Modified Grashof number 
Figure 7, shows the influence of Modified Grashof number on velocity profile of the fluid while holding the other parameters constant. The graph of the velocity value is plotted by taking the distance from moving channel wall to stationary channel wall denoted by $y$ along the horizontal axis while taking the value of the fluid velocity denoted by $u$ along vertical axis.

The result shows that for a fixed value of Modified Grashof number $G c$, the value of the fluid velocity denoted by $u$ starts from a minimum constant value at the moving channel wall, and as $y$ increases the velocity $u$ increases and reaches a maximum constant value at the stationary channel wall. Generally, we can conclude from the simulation study that velocity of the fluid increases as modified Grashof number increases.

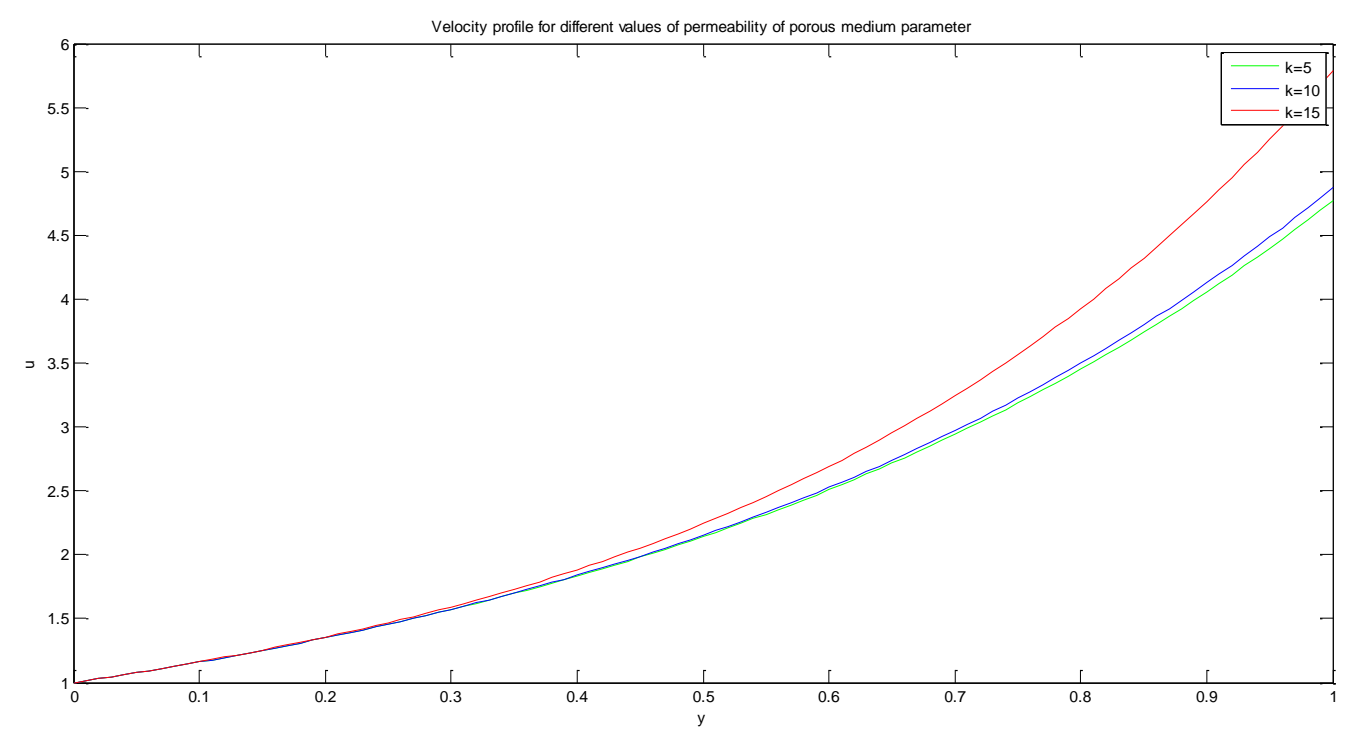

Figure 8. Velocity profile of the model for different values of permeability of porous medium parameter

Figure 8, shows the influence of permeability of porous medium on velocity profile of the fluid while holding the other parameters constant. The graph of the velocity value is plotted by taking the distance from moving channel wall to stationary channel wall denoted by $y$ along the horizontal axis while taking the value of the fluid velocity denoted by $u$ along vertical axis.

The result shows that for a fixed value of permeability of porous medium, the value of the fluid velocity denoted by $u$ starts from a minimum constant value at the moving channel wall, and as $y$ increases the velocity $u$ increases and reaches a maximum constant value at the stationary channel wall. Generally, we can conclude from the simulation study that as permeability of porous medium increases the velocity of the fluid increases. Physically, this means that the resistance dominated by the porous medium reduces as the permeability of the medium increases because of which the velocity increases. 


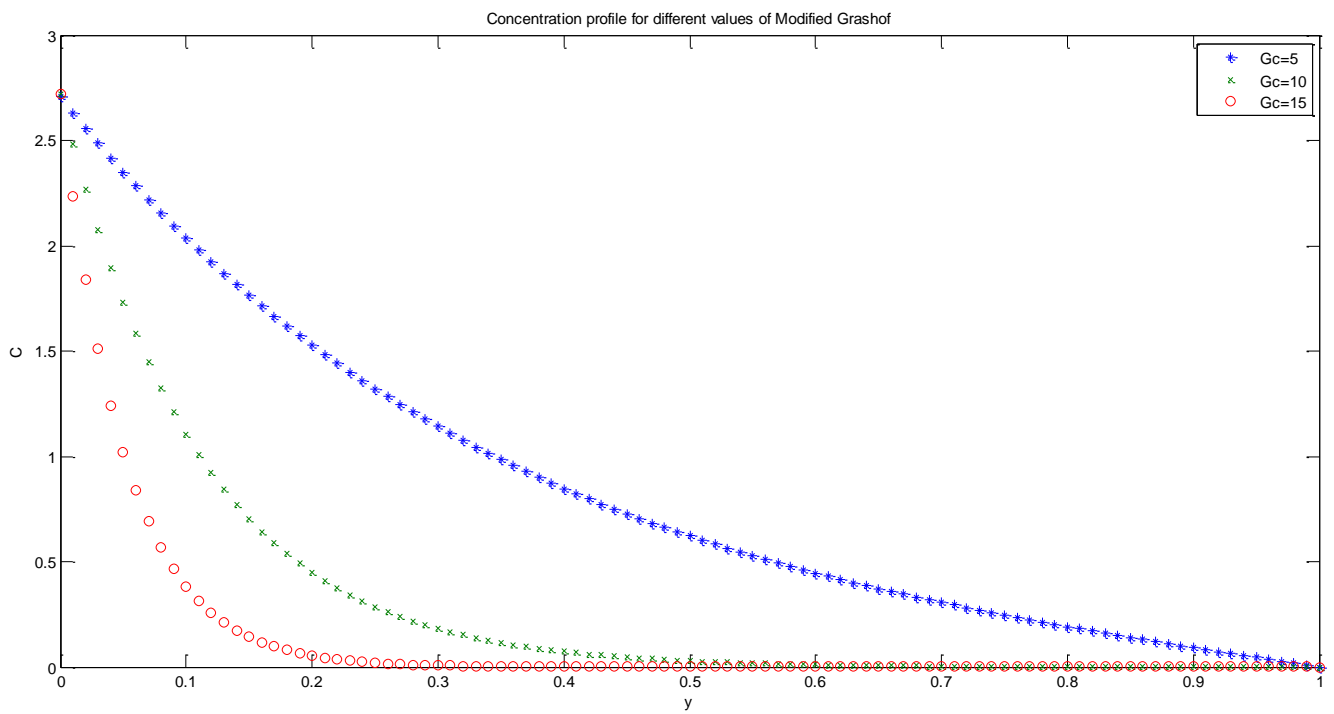

Figure 9. Concentration profile of the model for different values of Modified Grashof number

Figure 9, the influence of Modified Grashof number on the concentration of the fluid has been studied by simulation. Naturally, the number and the concentration are inversely proportional to each other i.e.as Modified Grashof number increases the velocity of the fluid increases and hence the concentration decreases. The same fact has been even proved by the present simulation study.

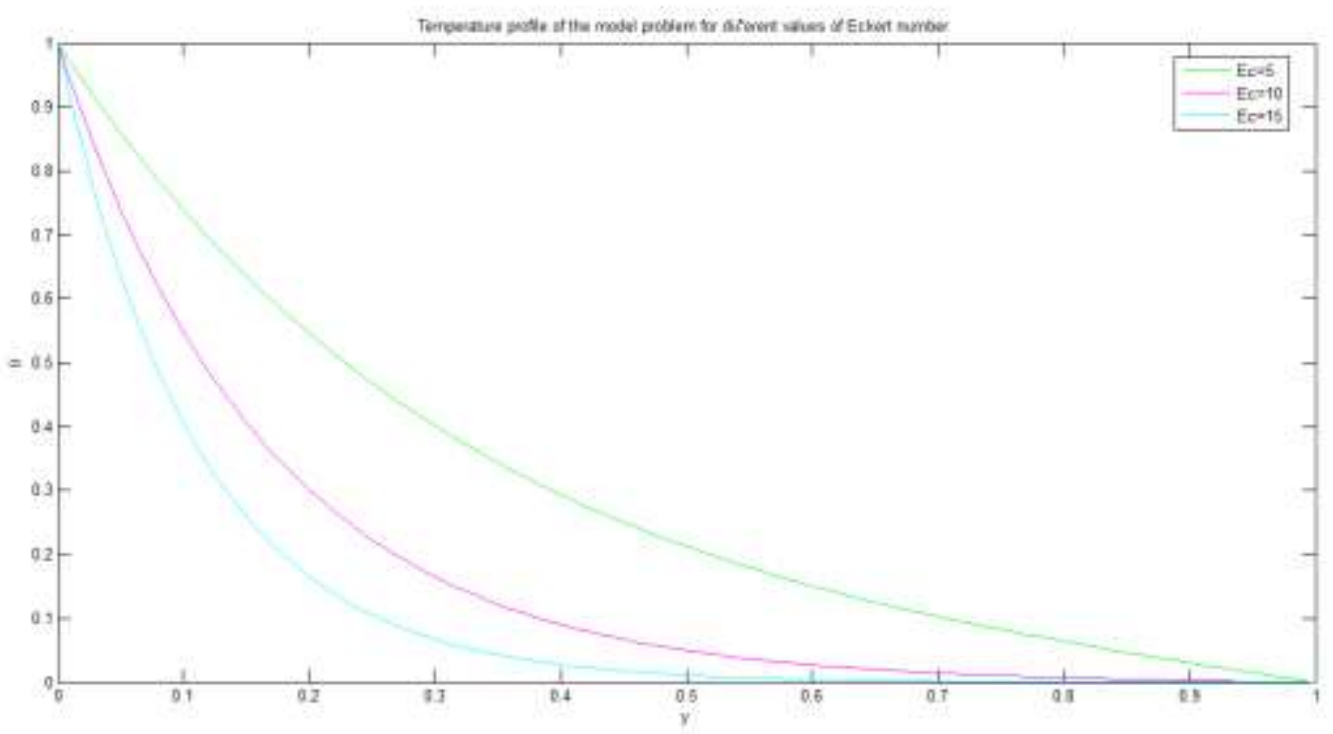

Figure 10. Temperature profile of the model for different values of Eckert number

Figure 10, shows the influence of Eckert number on transfer of the temperature while holding the other parameters constant. The graph of the temperature is plotted by taking the 
distance from moving channel wall to stationary channel wall denoted by $y$ along the horizontal axis while taking the value of temperature denoted by $\theta$ along vertical axis.

From the simulated graph it can be conclude that as the Eckert number increases the temperature decreases. Physically this shows how kinetic energy can be lost to internal energy by deformation of fluid elements.

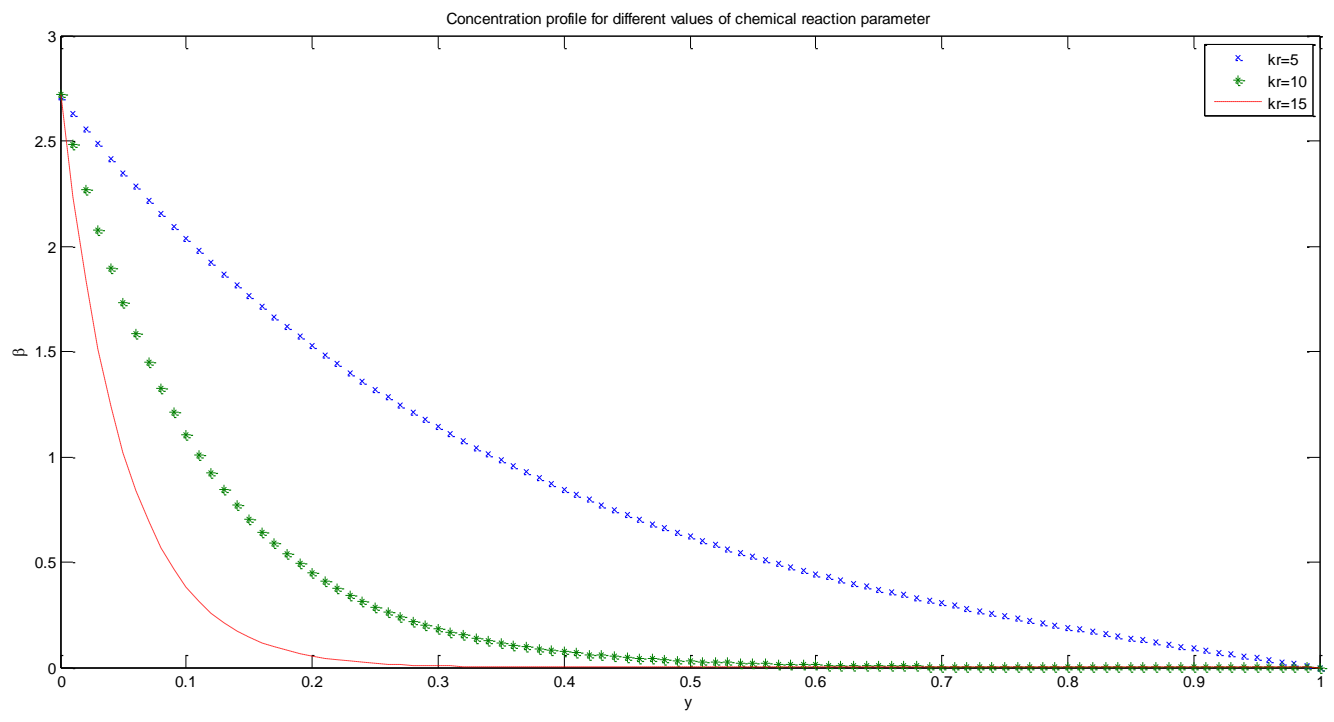

Figure 11. Concentration profile of the model for different values of chemical reaction parameter

Figure 11, shows the influence of Chemical reaction parameter on concentration profile of the fluid while holding the other parameters constant. The graph of the concentration value is plotted by taking the distance from moving plate to stationary channel wall denoted by $y$ along the horizontal axis while taking the value of fluid concentration denoted by $\beta$ along vertical axis

The result shows that for a fixed value of chemical reaction parameter, the value of fluid concentration denoted by $\beta$ starts from a maximum constant value at the moving, channel wall and as $y$ increases the concentration $\beta$ decreases and reaches zero constant value at the stationary channel wall. Nevertheless, the measure of fluid concentration at the moving channel wall is always at a higher value than that at the stationary channel wall.

As the concentration of fluid at the moving channel wall is higher than that at the stationary channel wall, the concentration is expected to move from moving channel wall towards stationary channel wall. Fluids diffuse from the locations of higher concentrations to that of lower concentrations. This fact has been proved in this simulation.

Also it can be observed from that simulation: (i) the value of concentration of fluid does not change with the change in chemical reaction parameter at the moving channel wall, (ii) the value of concentration of fluid, occurring between the two, channel walls decreases with the increase of chemical reaction parameter i.e. the value of concentration of fluid is inversely proportional to that of chemical reaction parameter, and (iii) the value of concentration of fluid 
goes to zero at the stationary channel wall irrespective of the value of chemical reaction parameter.

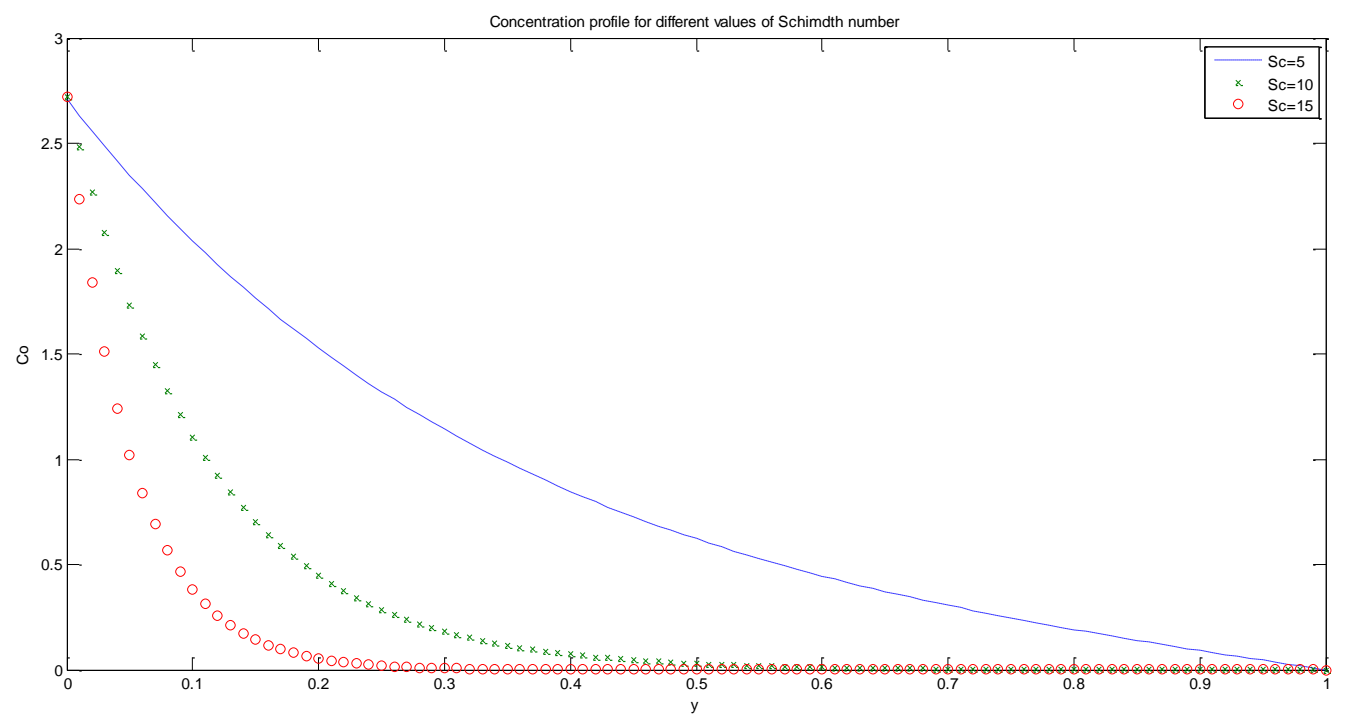

Figure 12. Concentration profile of the model for different values of Schmidt number

Figure 12, shows the influence of Schmidt number on concentration profile of the fluid while holding the other parameters constant. The graph of the concentration value is plotted by taking the distance from moving channel wall to stationary channel wall denoted by $y$ along the horizontal axis while taking the value of fluid concentration denoted by co along vertical axis.

The result shows that for a fixed value of Schmidt number the value of fluid concentration denoted by co starts from a maximum constant value at the moving channel wall, and as $y$ increases the concentration $c o$ decreases and reaches zero constant value at the stationary channel wall. Nevertheless, the measure of fluid concentration at the moving channel wall is always at a higher value than that at the stationary channel wall.

As the concentration of fluid at the moving plate is higher than that at the stationary channel wall, the concentration is expected to move from moving channel wall towards stationary channel wall. Fluids diffuse from the locations of higher concentrations to that of lower concentrations. This fact has been verified in this simulation.

Also it can be observed from that simulation: (i) the value of concentration of fluid does not change with the change in Schmidt number at the moving channel wall, (ii) the value of concentration of fluid, occurring between the two channel walls, decreases with the increase of Schmidt number i.e. the value of concentration of fluid is inversely proportional to that of Schmidt number, and (iii) the value of concentration of fluid goes to zero at the stationary channel wall irrespective of the value of Schmidt number.

In general, the concentration decreases with an increase in Schmidt number. Physically, this is true since the increase in Schmidt number implies decrease of molecular diffusivity implies decrease in concentration of the boundary layer. 


\section{Conclusion}

In this paper unsteady, incompressible, magneto hydrodynamics with electrically conducting viscoelastic fluid in an infinite vertical Couette channel walls embedded in a porous medium has been analyzed. The effects of physical parameters Viz. Hartmann number, Viscoelastic parameter, Permeability of porous medium, Chemical reaction parameter, thermal Grashof number for heat transfer, modified Grashof number for mass transfer, frequency parameter and Schmidt number on flow variables Viz. velocity, temperature and concentration has been discussed. The solution of the governing equations is obtained using regular perturbation techniques. These techniques are used to transform partial differential equations that are difficult to solve in closed form or explicit form. MATLAB code is used to solve higher order ordinary differential equations and to simulate the graphs. Hence the following results are obtained

i. Velocity decreases with increasing values of frequency, Hartmann number, Viscoelastic parameter.

ii. Velocity increases with temperature, thermal Grashof number, modified Grashof number and permeability of porous medium.

iii. An increment in both thermal radiation parameter and Eckert number results in decrement of temperature near the moving porous channel wall while it approaches to a zero in the region close to the boundary layer of the stationary channel wall,

iv. An increment in both chemical reaction and Schmidt number results in decreasing in concentration.

v. With the development of modern industry the importance of using non-Newtonian fluid such as lubricants has been emphasized. Common lubricants exhibiting non-Newtonian behavior are polymer, oils and greases.

vi. The effects of coupled stress and permeability in free convection heat transfer in fluid flows have been analyzed.

vii. Since the radiative parameter and temperature are inversely proportional. This can be applied in bio medical engineering such as radiotherapy, CT scan, Doppler ultrasound, MRI.

viii. Viscoelastic fluid in the presence of thermal radiation, chemical reaction, viscous dissipation and permeability of porous medium has been analyzed.

\footnotetext{
Nomenclature

$\mathrm{Gr}$ - thermal Grashof number

Gc - modified Grashof number

$S c-$ Schmidt number

$t$ - time

$M$ - Hartmann number

Pr - Prandtl number

$q_{r}$ - radiative heat flux

$k_{0}-$ Viscoelastic parameter

$E_{c}-$ Eckert number

$k_{r}$ - chemical reaction parameter

$R$ - radiation parameter
} 
$D$ - mass diffusivity

$T^{\prime}$ - temperature of the fluid in the boundary layer

$T^{\prime}{ }_{w}$ - temperature of the moving channel wall

$T^{\prime}{ }_{h}$ - temperature of the stationary channel wall

$U^{\prime}-$ free stream velocity

$U-$ dimensionless free stream velocity

$u^{\prime}$ - velocity component in $x^{\prime}$ direction

$v^{\prime}$-velocity component in $y^{\prime}$ direction

$x^{\prime}, y^{\prime}-$ Cartesian coordinates

$x, y-$ dimension less Cartesian coordinates

$C^{\prime}{ }_{w}$ - concentration at channel wall at $y=0$

$C_{h}^{\prime}$ - concentration at channel wall at $y=h$

$C$ - dimension less concentration

$C_{p}-$ Specific heat at constant pressure

$J$ - Electric current density

$b_{1}$ - Induced magnetic field

$B_{0}-$ Strength of magnetic field

$g-$ acceleration due to gravity

$\beta-$ thermal expansion coefficient

$\beta_{c}-$ concentration expansion coefficient

$\varepsilon-$ amplitude of free stream velocity

$\omega-$ frequency of oscillation

$\theta-$ dimensionless temperature

$\kappa-$ thermal conductivity

$v-$ kinematic viscosity

$\mu-$ dynamic viscosity

$\alpha-$ thermal diffusivity

$\sigma-$ electric conductivity.

\section{References}

[1] K. Walters, Non-Newtonian effects in some elastic-viscous liquids whose behavior at small rates of shear is characterized by a general linear equation of state. Quant. J. Mech. Appl. Math, 15 (1962) 63-76.

[2] Bhaskara Reddy, N. and Bathaiah, D. Reg. J. of Energy Heat Mass Transfer, 3, No.4, (1981) 239-255.

[3] Bhaskara Reddy, N. and Bathaiah, D. Acta Mechanica, 42, (1982) 239-251.

[4] Chowdhury, M. K. and Islam, M. N. MHD free convection flow of viscoelastic fluid past an infinite porous plate, Int. J. Heat Mass Trans.,36 (2000) 439-447.

[5] Rajgopal, K., Veena, P.H. and Parvin, V.K. Oscillatory Motion of an Electrically Conducting Viscoelastic Fluid over a Stretching Sheet in Saturated Porous Medium with Suction/Blowing. Mathematical Problems in Engineering, 1(2006) 1-14.

[6] P.R. Sharma and D. Pareek, Unsteady flow and heat transfer through an elastic viscous liquid along an infinite hot vertical porous moving plate with variable free stream suction. Bull. Cal. Math. Sec. 98 (2006) 97-108. 
[7] Pravat Kumar Rath, G.C. Dash, and P.K. Rath, Flow and heat transfer of an electrically conducting viscoelastic fluid between two horizontal squeezing/stretching plates. AMSE Modeling Measurement and Control, 70 (2001) 45-63

[8] S.M.B., Alhari, A.A. Mohamed and M. S.E.L. Gerdy, Heat and Mass transfer in MHD viscous elastic fluid flow through a porous medium over a stretching sheet with chemical reaction. Applied Mathematics, 1(2010) 446-455

[9] B. Kumar and R. Sivaraj, MHD mixed convective viscoelastic fluid flow in a permeable vertical channel with Dufour effect and chemical reaction, Int. J. of Appl. Math. And Mech, 14 (2011) 79-96.

[10] R.A. Damesh and B.A. Shannak, Viscoelastic fluid flow past infinite vertical porous plates in the presence of first order chemical reaction. Int.J. of Appl. Math. Mech. Engl. Ed. 31 (2010) 955-962

[11] S. Dash, G.C. Dash and D.P. Mishra, MHD flow through a porous medium past a stretched vertical permeable surface in the presence of heat source/sink and a chemical reaction. Proc. Nat. Acad. Sci. India., 78A (2008) 49-55.

[12] P.K. Rath, T. Parida and G.C. Dash, Three-Dimension free convective flow through porous medium in a vertical channel with heat source and chemical reaction. Proc. Nat. Acad. Sci. India, 82A (2012) 225-232.

[13] P.R. Sharma and S. Sharma, Unsteady two dimensional flow and heat transfer through an elastic viscous liquid along an infinite hot vertical porous surface bounded by porous medium. Bull. Cal. Math. Sec. 97 (2005) 477-488.

[14] Arpita Mohanty, Pravat Kumar Rath, G.C.Dash, Unsteady MHD flow of a viscoelastic fluid a long a vertical porous surface with fluctuating temperature and concentration IOSR Journal of Engineering, ISSN (e); 2250-3021, ISSN (p); 2278-8719, 4, (2014) 4657

[15] Pawan Kumar Sharma, Mukesh Dutt, MHD oscillatory free convection flow past parallel plates with periodic temperature and concentration, Universal Journal of Applied Mathematics, 2,(2014) No.7,264 - 75, Doi: 10.13189/ujam.2014,020702

[16] T. Sarpkaya, Flow of non-Newtonian fluids in a magnetic field, AICHE Journal,7(1961), 324- 328.

[17] Cogley, A.C., Vinceti, W.C. and Gilles, S.E., Differential Approximation for Radiation Transfer in a Nongray Gas near Equilibrium. American Institute of Aeronautics and Astronautics Journal, 6(1968) 551-555

[18] Mansour, M.A., Radiative and Free Convection Effects on the Oscillatory Flow past a Vertical Plate. Astrophysics and Space Science, .166(1990), 269-275

[19] Hossain, M.A. and Thakar, H.S., Radiation Effect on Mixed Convection along a Vertical Plate with Uniform Surface Temperature. Heat and Mass Transfer, 314(1996) 243-248

[20] Hossain, M.A., Alim, M.A. and Rees, S, The Effect of Radiation on Free Convection from a Porous Vertical Plate, International Journal of Heat and Mass Transfer,42(1999) 181191

[21] Muthucumarswamy, R. and Senthil, G.K, Studied the Effect of Heat and Mass Transfer on Moving Vertical Plate in the Presence of Thermal Radiation, Journal of Theoretical and Applied Mechanics,31(2004) 35-46.

[22] Aydin, A. and Kaya, A., Radiation Effect on MHD Mixed Convection Flow about a Permeable Vertical Plate. Heat and Mass Transfer, 45(2008) 239-246. 
[23] Muthucumaraswamy, R. and Janakiraman, B., Mass Transfer Effects on Isothermal Vertical Oscillating Plate in the Presence of Chemical Reaction. International Journal of Applied Mathematics and Mechanics, 4 (2008) 66-74.

[24] Sudheer Babu, M. and Satya Narayan, P.V, Effects of the Chemical Reaction and Radiation Absorption on Free Convection Flow through Porous Medium with Variable Suction in the Presence of Uniform Magnetic Field. Journal of Heat and Mass Transfer, 3,(2009) 219-234.

[25] Makinde, D. and Chinyoka, T, Numerical Study of Unsteady Hydromagnetic Generalized Couette Flow of a Reactive Third-Grade Fluid with Asymmetric Convective Cooling. Computers and Mathematics with Applications, 61(2011) 1167-1179.

[26] Skelland A.H.P, Non-Newtonian Flow and Heat Transfer, John Wiley, Sons, New York, (1976).

[27] Brewster M.A, Thermal Radiative Transfer and Properties. - New York: John Wiley and Sons (1992).

[28] B.zigta and P.R.Koya, The Effect of MHD on Free Convection with periodic Temperature and Concentration in The Presence of Thermal Radiation and Chemical reaction. International Journal of Applied Mechanics and Engineering,.22 (2017), No.4,1059-1073 DOI: 10.1515/ijame-2017-0068.

[29] B. Zigta, The Effect of Thermal Radiation, Chemical reaction and Viscous Dissipation on MHD Flow. International Journal of Applied Mechanics and Engineering, 23, No.3, (2018)787-801 DOI: 10.2478/ijame-2018-0043.

[30] Olanrewaju PO, Alao FI, Adeniyan A, Effects of thermal diffusion, diffusion thermo, magnetic field and viscous dissipation on unsteady mixed convection flow past a porous plate moving through a binary mixture of chemically reacting fluid. Thermo Energy Power Eng, 2(2013) 134-46.

[31] G.V. Ramana Reddy, N. Bhasakar Reddy and R.S.R. Gorla, Radiation and Chemical Reaction Effects on MHD Flow along a Moving Vertical Porous Plate. Int. J. of Applied Mechanics and Engineering, 2016, .21, (2016) No.1, 157-168 DOI: 10.1515/ijame-20160010

[32] Basant K. Jha, B.Y. Isah, I.J. Uwanta, Unsteady MHD free convective Couette flow between vertical porous plates with thermal radiation, Journal of King Saud UniversityScience, 27, (2015) 338-348

\section{APPENDIX}

$$
\begin{aligned}
& m= \pm \sqrt{\frac{k_{r} h^{2}}{D}}, \quad \gamma= \pm \sqrt{\frac{k_{r} h^{2}}{D}+i \omega S c, \quad \lambda}= \pm i \sqrt{3 Q_{0} h^{2} \operatorname{Pr} / \rho C_{p} v(4 R+3)} \\
& \mu= \pm i \sqrt{3 \operatorname{Pr}\left(Q_{0} h^{2}-i \omega \rho C_{p} v\right) / \rho C_{p} v(4 R+3)} \\
& c_{1}=\frac{1}{e^{2 \lambda}-1}, \quad c_{2}=\frac{e^{2 \lambda}}{e^{2 \lambda}-1}, \quad d_{1}=\frac{-1}{e^{2 m}-1}
\end{aligned}
$$


Budapest International Research in Exact Sciences (BirEx) Journal

Volume 1, No 3, July 2019, Page: 35-57

e-ISSN: 2655-7827 (Online), p-ISSN: 2655-7835 (Print)

www.bircu-journal.com/index.php/birex

emails: birex.journal@gmail.com

birex.journal.qa@gmail.com

$$
\begin{aligned}
& d_{2}=\frac{e^{2 m}}{e^{2 m}-1}, \quad h_{1}=\frac{2-e^{2 \gamma}}{1-e^{2 \gamma}}, \quad h_{2}=\frac{-1}{1-e^{2 \gamma}}, k_{1}=\frac{-1}{e^{2 \mu}-1}, \quad k_{2}=\frac{e^{2 \mu}}{e^{2 \mu}-1} \\
& \phi=\frac{\alpha b^{2}}{v \mu \omega}+\frac{U_{0}^{2} \alpha b^{2}}{k_{0} v^{2} \mu^{2} \omega} \quad, \psi=\frac{U_{0}^{4} u_{0} \alpha b^{4}}{\kappa v^{4} \mu^{2} \omega k_{0}} \quad, \quad \chi=\frac{U_{0}^{2} \alpha b^{2}}{k_{0} v^{2} \mu^{2} \omega}, \\
& \Omega=\frac{\alpha b^{2}}{i v \mu \omega}+\frac{U_{0}{ }^{2} \alpha b^{2}}{i k_{0} \nu^{2} \mu^{2} \omega}, \Gamma=\frac{U_{0}{ }^{4} u_{0} \alpha b^{4}}{i \kappa v^{4} \mu^{2} \omega k_{0}}, K=\frac{U_{0}{ }^{2} \alpha b^{2}}{i k_{0} \nu^{2} \mu^{2} \omega}
\end{aligned}
$$

